\title{
Correction to: Overview of therapeutic plasma exchange in pediatric neurology: a single-center experience
}

\author{
Murat Özkale $^{1} \cdot$ Ilknur Erol $^{2} \cdot$ Yasemin Özkale ${ }^{1} \cdot$ Illknur Kozanoğlu ${ }^{3}$
}

Published online: 28 June 2018

(c) Belgian Neurological Society 2018

\section{Correction to: Acta Neurologica Belgica https://doi.org/10.1007/s13760-018-0961-5}

Unfortunately, the immediate following sentences were incorrectly published in Material and Method section of the original article.

A venous catheter was placed into the internal jugular or femoral veins. TPE was carried out at the predicted 1-1.5 $\times$ plasma volume every other day using Fresenius Com Tec apheresis devices (Fresenius AG, Germany). TPE was performed using the centrifugal filtration method in our center.
The complete corrected sentences are given below.

A venous catheter was placed into the internal jugular or femoral veins. TPE was carried out at the predicted $1-1.5 \times$ plasma volume every other day using Spectra Optia v11.3 (Terumo BCT Inc., Lakewood, CO, USA). TPE was performed using continuous centrifugation method in our center.

The original article can be found online at https://doi.org/10.1007/ s13760-018-0961-5.

Yasemin Özkale

dryaseminozkale@gmail.com

1 Department of Pediatrics, Baskent University Faculty of Medicine, Adana Dr Turgut Noyan Teaching and Medical Research Center, Baraj Yolu 1 Durak, Seyhan, 01120 Adana, Turkey

2 Department of Pediatric Neurology, Baskent University Faculty of Medicine, Dr Turgut Noyan Teaching and Medical Research Center, Adana, Turkey

3 Department of Physiology, Baskent University Faculty of Medicine, Dr Turgut Noyan Teaching and Medical Research Center, Adult Bone Marrow Transplantation Center, Apheresis Unit, Adana, Turkey 